\title{
AN ON-CHIP AUTONOMOUS MICROFLUIDIC COOLING SYSTEM
}

\author{
Abhishek K. Agarwal ${ }^{1}$, Sudheer S. Sridharamurthy ${ }^{1}$, David J. Beebe ${ }^{2}$, and Hongrui Jiang ${ }^{1, *}$ \\ ${ }^{1}$ Department of Electrical and Computer Engineering, University of Wisconsin-Madison, Madison, WI, USA \\ ${ }^{2}$ Department of Biomedical Engineering, University of Wisconsin-Madison, Madison, WI, USA \\ * E-mail: hongrui@engr.wisc.edu
}

\begin{abstract}
An on-chip self-contained autonomous microfluidic cooling system, driven here by a constant external rotating magnetic stirrer, has been developed using liquid-phase photopolymerization and nickel electroplating. A temperature-sensitive hydrogel that acts similar to an automotive clutch provides the autonomous functionality. By controlling the rotation of the nickel impeller, the hydrogel effectively controls the pumping of cold water to cool the system when temperatures are high. Once cooled, the system autonomously stops pumping. The autonomous functionality and cooling effect of the system are observed at various heater temperature setpoints. Cooling temperatures ranging between $1.6^{\circ} \mathrm{C}$ and $4.0^{\circ} \mathrm{C}$ were exhibited by the system.
\end{abstract}

Keywords: autonomous, magnetic actuator, microfluidic, micropump, on-chip cooling

\section{INTRODUCTION}

Thermal management is of crucial concern in microelectronics [1] and recently, biological microfluidics, e.g., polymerase chain reaction (PCR) and DNA analysis [2]. Many existing varieties of thermal management exist, including air jet flow, dielectric fluids, fans, cold plates, and refrigeration systems [1]. However, a majority of these systems are bulky, demand high power, and are not easily scaled down and/or integrated into devices at the microscale $[1,3,4]$.

We present a first demonstration of an on-chip selfcontained autonomous microfluidic cooling system. It actively responds to local temperatures on-chip and does not require additional temperature sensing, feedback and control, or pressure flow.

The autonomous cooling is realized through a programmable clutch mechanism we previously reported $[5,6]$. The microfluidic cooling system is fabricated using a combination of microfluidic tectonics [7], specifically liquid-phase photopolymerization $\left(\mathrm{LP}^{3}\right)$ and nickel (Ni) electroplating. A temperature-responsive hydrogel ring is photopatterned around the center axis of a Ni impeller (Fig. 1 (b)) using LP $^{3}$. The hydrogel actively responds to its local temperature changes onchip by expanding and contracting in volume, acting as an autonomous clutch to turn the cooler on/off (Fig. 1 (c)). The hydrogel's responding temperature can be pre-
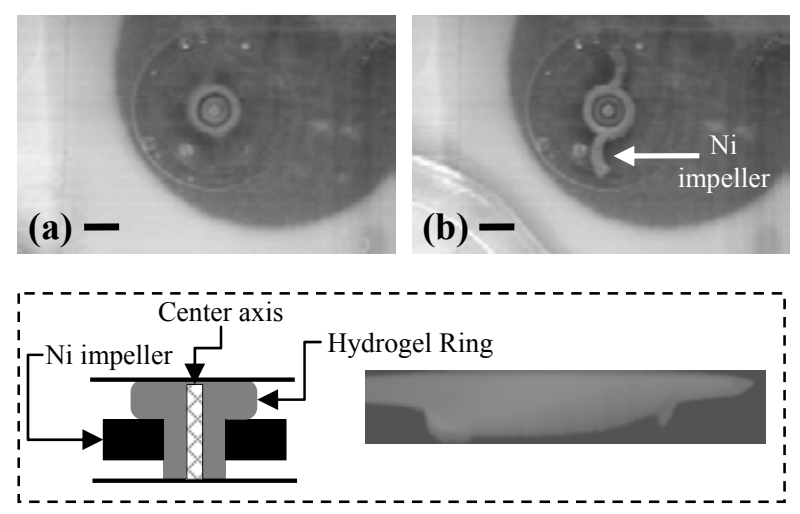

(c)

Fig. 1. Functional mechanism of the autonomous microfluidic cooling system as an effect of local temperature. The system is driven by a constant external rotating magnetic stirrer. Scale bars represent $1.25 \mathrm{~mm}$. (a) At high local temperatures, the temperature-responsive ring contracts, allowing the impeller to rotate. (b) At low local temperatures, the temperatureresponsive ring expands into a mushroom cap, constricting the Ni impeller. (c) Cross-section (left) of an expanded hydrogel ring. A similar cross-sectional confocal image of an expanded hydrogel (right) provides verification of the mushroom cap.

programmed by tuning its chemistry. When local temperatures rise above a pre-programmed value (here, $\sim 30^{\circ} \mathrm{C}$ ), the impeller autonomously responds by pumping and recirculating (cold) water until the local temperature decreases to a designed lower temperature (here, $26{ }^{\circ}$ C). See Fig. 1 (a) and (b).

\section{FABRICATION PROCESS}

The fabrication process is modified from previous work $[5,6]$ and is extended from two to three construct layers. The process utilizes $\mathrm{LP}^{3}$ of photosensitive polymers and $\mathrm{Ni}$ electroplating. High resolution (3600 dpi) film photomasks (Silverline Studio, Madison, WI, USA) are used to transfer patterns to the photosensitive polymers.

\section{Photosensitive Polymers}

Two photosensitive polymers, similar to negative photoresists, are used to create the structural (channels and posts) and functional (autonomous functionality) components of the cooling system.

The non-responsive photosensitive polymer used here to form channels and posts consists of three

\section{TRANSDUCERS'05}

The 13th International Conference on Solid-State Sensors, Actuators and Microsystems, Seoul, Korea, June 5-9, 2005 


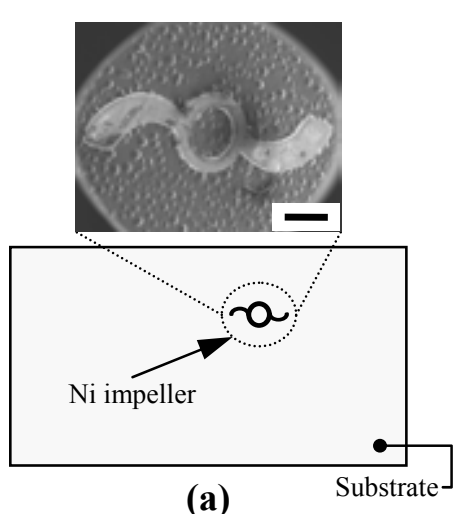

(a)

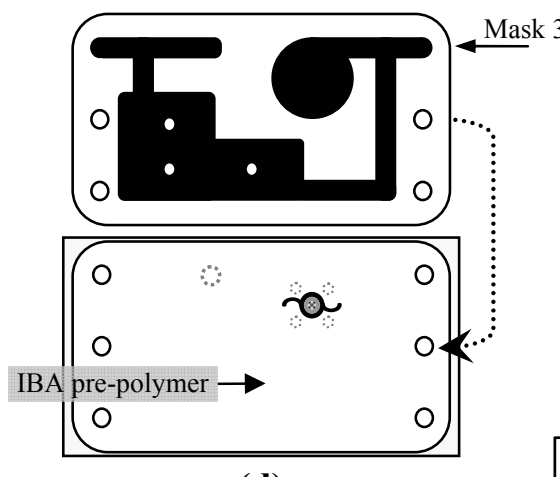

(d)

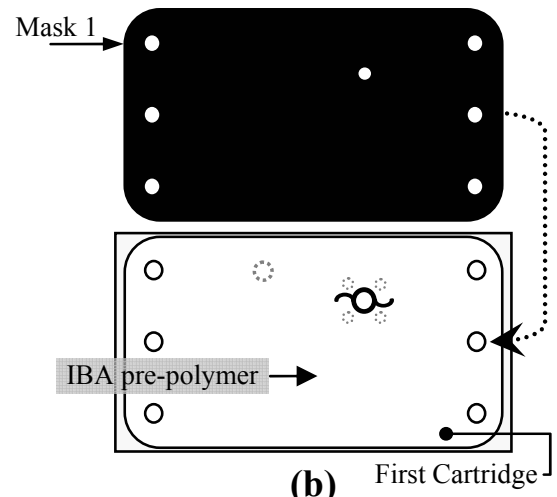

(b)

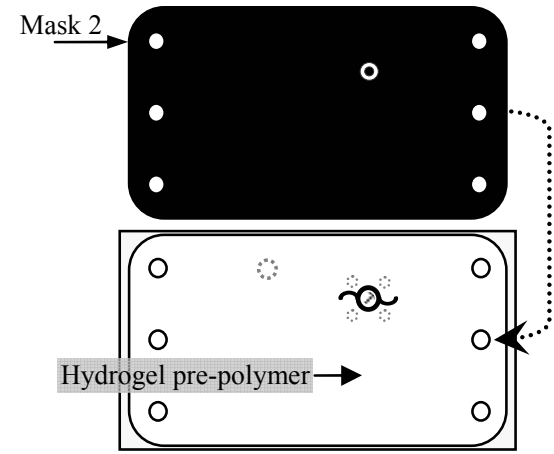

(c)

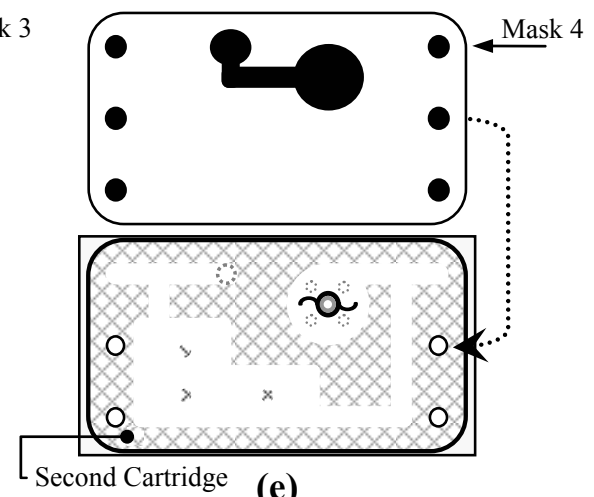

(e)
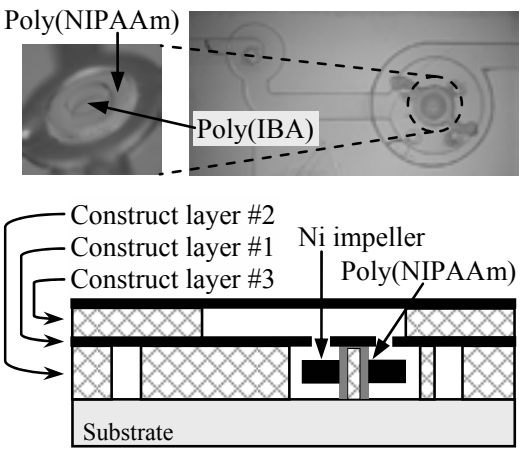

(f)

Fig. 2. Process for fabricating an on-chip three construct layer autonomous microfluidic cooling system. (a) A Ni impeller is previously electroplated on a microscope glass slide using photopatterned poly(IBA) as the defining mold [5, 6]. A SEM image of the impeller is shown above (scale bar represents $1.0 \mathrm{~mm}$ ). (b) A $250-\mu \mathrm{m}$ polycarbonate cartridge (construct layer \#1) is affixed to the substrate. A central core poly(IBA) post is photopatterned as the center axis of the impeller. (c) The temperature-responsive hydrogel ring (poly(NIPAAm)) is photopatterned around the poly(IBA) post, acting as a jacket. (d) The channels, pumping chamber, and cold water reservoir (construct layer \#2) are photopatterned using poly(IBA). (e) A second cartridge (125 $\mu \mathrm{m})$ is affixed on top of the first cartridge. The connecting channel is photopatterned with poly(IBA), forming construct layer \#3. (f) A cross-section of the three-layer autonomous micro cooling system. The two images above show the connection between construct layers \#2 and \#3 via the drilled holes in construct layer \#1 (right) as well as a zoom-in on the autonomous clutch (left).

constituents: monomer - isobornyl acrylate (IBA), crosslinker - tetraethylene glycol dimethacrylate (TeGDMA), and photoinitiator - 2,2-dimethoxy-2phenylacetophenone (DMPA). Exposure to a ultraviolet (UV) light source causes the pre-polymer solution to harden (called poly(IBA)). It is also used as a mold for Ni electroplating.

The temperature-responsive hydrogel pre-polymer consists of five constituents: temperature-sensitive monomer - $N$-isopropylacryamide (NIPAAm), N,N'methylenebisacrylamide (NMBA), photoinitiator DMPA, dimethyl sulphoxide (DMSO), and deionized (DI) water. Once photopatterned by UV light, the hydrogel chemistry used here results in a volume transition point, known as the lower critical solution temperature $(\mathrm{LCST})$, of $30^{\circ} \mathrm{C}$.

\section{Fabrication}

Devices are fabricated on microscope glass slides $(76.2 \mathrm{~mm} \times 25.4 \mathrm{~mm} \times 1 \mathrm{~mm})$ that have been previously coated with $\mathrm{Ti} / \mathrm{Cu} / \mathrm{Ti}(0.06 / 0.35 / 0.06 \mu \mathrm{m})$ using a DC sputterer. A poly(IBA) masking mold of the impeller is photopatterned on the glass slide. Double-sided adhesive tapes are used to create a cavity between the glass slide and photomask of the impeller. The IBA-based prepolymer is squeezed into the cavity using transfer pipettes and subsequently exposed to UV light (intensity, $I=7.7 \mathrm{~mW} / \mathrm{cm}^{2}$; time, $\left.t=18.5 \mathrm{~s}\right)$. The photomask is carefully removed and the device is developed in a bath of $100 \%$ ethyl alcohol for $180-240 \mathrm{~s}$. Next, it is softbaked on a hotplate for $15 \mathrm{~min}$ at $50{ }^{\circ} \mathrm{C}$.

The top Ti layer is removed using 1:10 $\mathrm{HF}: \mathrm{H}_{2} \mathrm{O}$. Ni electroplating (Microfab $^{\circledR}$ NI 100, Enthone-OMI, Inc., West Haven, CT, USA) is performed at the active sites where $\mathrm{Cu}$ has been exposed using a current density of $5 \times 10^{-4} \mathrm{~A} / \mathrm{mm}^{2}$ [8] to create an extruded 2.5-dimensional Ni impeller. The poly(IBA) masking mold is removed after immersion in a 3:1 methanol:acetone bath for 
several hours. The seed metal layers are also removed, using 1:1:10 HAC: $\mathrm{H}_{2} \mathrm{O}_{2}: \mathrm{H}_{2} \mathrm{O}$ to selectively etch $\mathrm{Cu}$ [9]. See Fig. 2 (a).

A polycarbonate cartridge (HybriWells ${ }^{\mathrm{TM}}$, Grace-Bio Labs, Inc., Bend, OR, USA) with a spacer layer of $250 \mu \mathrm{m}$ is affixed to the glass slide. This forms construct layer \#1. Inter-layer connection holes are previously drilled into the cartridge (dashed gray circles in Fig. 2 (b)). Next, construct layer \#2 is formed. The cartridge cavity is first filled with the IBA-based prepolymer and a poly(IBA) central core post (diameter $=$ $0.80 \mathrm{~mm}$ ) is similarly photopatterned (mask 1) as the center axis of the Ni impeller. Next, the temperatureresponsive hydrogel pre-polymer is flowed into the cartridge. A poly(NIPAAm) ring (width $=0.225 \mathrm{~mm}$ ) is photopatterned $\left(I=15 \mathrm{~mW} / \mathrm{cm}^{2}, t=10 \mathrm{~s}\right)$ from mask 2 , and subsequently rinsed with ethyl alcohol (Fig. 2 (c)). The Ni impeller is released by flowing in $\mathrm{Ti}$ and/or $\mathrm{Cu}-$ etching solutions into the cartridge.

Using mask 3 , the channels, pumping chamber, and cold water reservoir are photopatterned using poly(IBA), forming the rest of construct layer \#2 (Fig. 2 (d)). Before affixing the second cartridge $(125 \mu \mathrm{m}$ thick), construct layers \#1 and \#2 are filled with DI water to prevent backflow of the IBA-based pre-polymer in the subsequent step. The IBA-based pre-polymer is flowed into the second cartridge and the inter-layer connecting channel (construct layer \#3) is photopatterned $(I=$ $7.7 \mathrm{~mW} / \mathrm{cm}^{2}, t=16.5 \mathrm{~s}$ ) using mask 4. See Fig. 2 (e). A cross-section of the three-layer autonomous microfluidic cooling system is shown in Fig. 2 (f).

\section{EXPERIMENTS AND RESULTS}

Experiments were videotaped directly to a computer using a Sony Hyper HAD CCD-Iris/RGB camera (Sony Corp., New York City, NY, USA). An external rotating magnetic stirrer was used to drive the Ni impeller (Cole Parmer, model 84000-00, Vernon Hills, IL, USA).

\section{Experimental Setup}

The driving magnetic field's rotational frequency was set to $6.0 \mathrm{~Hz}$ for all experiments. See Fig. 3 (b) for experimental setup. A type- $\mathrm{K}$ thermocouple (model 5SRTC-TT-K-40-72, Omega Engr., Inc., Stamford, CT, USA), was inserted at the T-intersection in the channel network to monitor fluid temperature. To raise the temperature local to the hydrogel ring, a Kapton ${ }^{\mathrm{TM}}$ heater (model \#2-HK5537R7.8L12A, Minco Products, Inc., Minneapolis, MN, USA) was mounted on the underside of the glass slide using 3M \#9885 heat-conductive tape (3M, St. Paul, MN, USA). A temperature controller (CT16A3088-993, Minco Products, Minneapolis, MN, USA) was used to drive the Kapton ${ }^{\mathrm{TM}}$ heater. An onchip cold water reservoir kept below $20^{\circ} \mathrm{C}$ was provided.

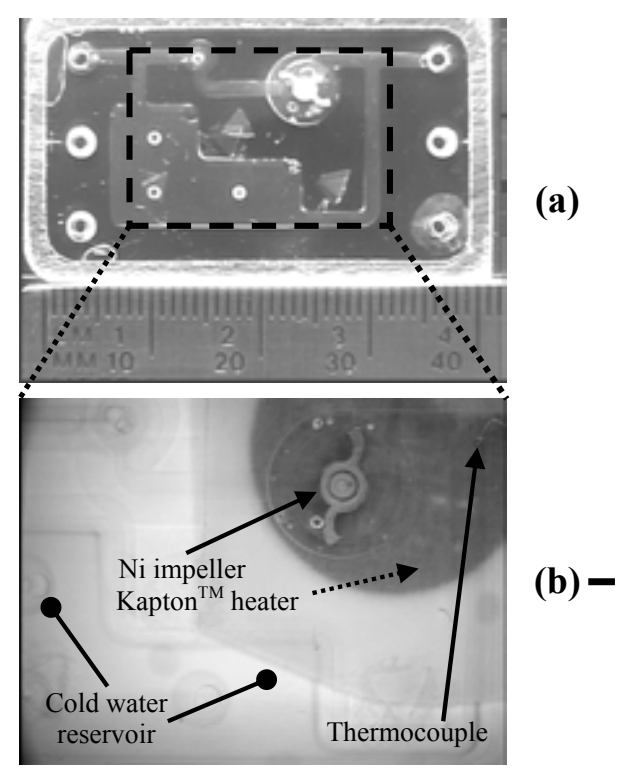

Fig. 3. Top-view photographs of (a) the complete autonomous microfluidic cooling system and (b) the experimental setup (zoomed-in). A type-K thermocouple is placed at the Tintersection to monitor fluid temperature. A Kapton ${ }^{\mathrm{TM}}$ heater is placed on the underside of the glass slide. The fluid is pumped clockwise. Scale bar represents $1.25 \mathrm{~mm}$.

\section{Autonomous Functionality}

First, the autonomous functionality of the cooling system was verified. See 'Run 1' in Fig. 4. Here, the rotating magnetic stirrer is constantly kept activated. The Kapton ${ }^{\mathrm{TM}}$ heater was initially turned on (setpoint $=$ $40{ }^{\circ} \mathrm{C}$ ), causing the $\mathrm{Ni}$ impeller to rotate and pump cold fluid autonomously. Once reaching the setpoint, the heater was turned off, allowing the system to cool from the pumping. Once the local fluid temperature dropped below $\sim 26^{\circ} \mathrm{C}$, the $\mathrm{Ni}$ impeller autonomously stopped rotating and pumping fluid. The heater was turned back on again, and when the temperature rose above $27-30{ }^{\circ} \mathrm{C}$, the Ni impeller started to rotate again and pump cold fluid. This on/off heater cycle was repeated four times to exhibit the autonomous functionality of the cooling system.

\section{Cooling Effect}

The cooling effect was observed by providing a constant heat influx into the device (mimicking heat sources on-chip) using the Kapton ${ }^{\mathrm{TM}}$ heater. The temperature controller works on a continuous feedback system provided by the thermocouple. To fool the system into inputting a constant heat flux, the feedback probe was kept $5 \pm 0.5{ }^{\circ} \mathrm{C}$ lower than the heater setpoint. A second thermocouple was used separately to monitor fluid temperature inside the system.

See Fig. 4 - 'Runs 2a-c'. Three separate heater setpoints of $27^{\circ} \mathrm{C}$ ('Run $2 \mathrm{a}$ '), $40^{\circ} \mathrm{C}$ ('Run 2b'), and 


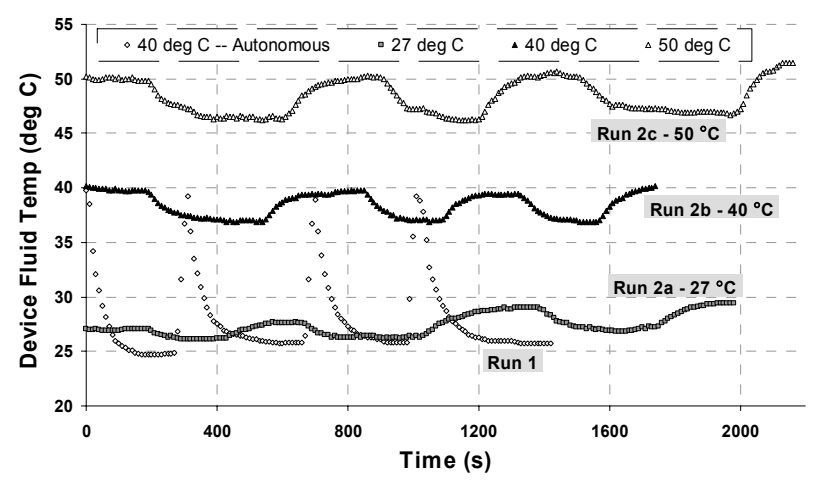

Fig. 4. Autonomous functionality and cooling effect experiments. 'Run 1' - the heater was turned on/off four times (setpoint $=40{ }^{\circ} \mathrm{C}$ ) to show the cooling pump autonomous functionality, i.e., at its lowest temperature in each of the four cycles, the $\mathrm{Ni}$ impeller stops rotating, despite the constant presence of a rotating magnetic field. The Ni impeller rotates again at the onset of temperatures above $30{ }^{\circ} \mathrm{C}$. 'Runs $2 \mathrm{a}-\mathrm{c}$ ' show the cooling effect of the system at heater setpoints 27,40 , and $50{ }^{\circ} \mathrm{C}$ whilst exposed to a constant heat influx source. Cooling repeatability, shown here by the cyclical troughs and crescents, was accomplished by turning the magnetic stirrer on/off three times.

$50{ }^{\circ} \mathrm{C}$ ('Run 2c') were tested. Cooling repeatability (shown here by the cyclical troughs and crescents) was observed by turning the magnetic stirrer on/off three times. Once the system reached a preset setpoint, the stirrer was activated until the fluid temperature lowered and stabilized. At this point, the magnetic stirrer was deactivated, allowing the fluid temperature to rise to the preset setpoint. This cycle was repeated three times for each heater setpoint. Local fluid temperatures decreased due to pumping and recirculation of cold water from the reservoir on-chip. At three different heater setpoints (27, 40 , and $50{ }^{\circ} \mathrm{C}$ ), the microfluidic cooling system repetitively lowered the temperature by an average of 1.6, 2.9, and $4.0^{\circ} \mathrm{C}$, respectively. Small fluctuations in the ambient room temperature caused the slight rise in the device fluid temperature in 'Run $2 \mathrm{a}$ '.

\section{CONCLUSION}

An on-chip self-contained autonomous microfluidic cooling system has been demonstrated here. The fabrication process is flexible to allow many substrates, including glass (used here), silicon, and silicon nitride/oxide. Furthermore, microfluidic cooler arrays can be fabricated where each cooler is programmed differently by altering the geometry and/or chemistry of the temperature-responsive hydrogel. The system demonstrated here is driven by a constant external rotating magnetic field. It is possible to integrate a local actuator, such as a three-phase micromotor [10]. The microfluidic cooling effect can be improved with an optimized $\mathrm{Ni}$ impeller design. Additionally, with different responsive hydrogels, the autonomous operation can be extended to different environmental parameters.

\section{Acknowledgments}

This work is supported by the Wisconsin Alumni Research Foundation (WARF). The authors would like to thank the Wisconsin Center for Applied Microelectronics (WCAM) (cleanroom processing) and Waisman Center (laser confocal imaging) at the University of Wisconsin-Madison. The authors are grateful to Richard Noll at the Materials Science Center at the University of Wisconsin-Madison for assistance in acquiring SEM image. The authors also recognize Drs. Javier Atencia and Liang Dong for technical discussions.

\section{References}

[1] A. Bar-Cohen, A. Watwe, and K.N. Seetharamu, "Fundamentals of thermal management"; pp. 213-217 in Fundamentals of Microsystems Packaging, Ed. R.R. Tummala, McGraw-Hill, London, 2001.

[2] A. Fuchs, H. Jeanson, P. Claustre, et al., "A silicon labon-chip for integrated sample preparation by PCR and DNA analysis by hybridization," Proc. of $2^{\text {nd }}$ annual Conf. on Microtechnologies in Medicine \& Biology, pp. 227-231, Madison, WI, USA, May 2-4, 2002.

[3] J. Darabi, M.M. Ohadi, and D. DeVoe, "An electrohydrodynamic polarizing micropump for electronic cooling," J. Microelectromech. Syst., 10, 98-106 (2001).

[4] E.N. Wang, L. Zhang, L. Jiang, J.-M. Koo, J.G. Maveety, E.A. Sanchez, K.E. Goodson, and T.W. Kenny, "Micromachined jets for liquid impingement cooling of VLSI chips," J. Microelectromech. Syst., 13, 833-842 (2004).

[5] A.K. Agarwal, S.S. Sridharamurthy, T.M. Pearce, G.A. Mensing, D.J. Beebe, and H. Jiang, "Magnetically-driven actuation using liquid-phase polymerization (LPP) and its application: A programmable mixer," Proc. of Solid State Sensor, Actuator, and Microsystem Workshop, pp. 121-124, Hilton Head Island, SC, USA, June 6-10, 2004.

[6] A.K. Agarwal, J. Atencia, D.J. Beebe, and H. Jiang, "Magnetically-driven temperature-controlled microfluidic actuators," Proc. of $1^{\text {st }}$ annual Conf. on INSS, pp. 51-55, Tokyo, Japan, June 22-23, 2004.

[7] D.J. Beebe, J.S. Moore, Q. Yu, R.H. Liu, M.L. Kraft, B-H Jo, and C. Devadoss, "Microfluidic tectonics: A comprehensive construction platform for microfluidic systems," PNAS, 97(25), 13488-13493 (December 5, 2000).

[8] Microfab $^{\circledR}$ NI 100 data sheet, July 2000, Enthone-OMI Inc., West Haven, CT, USA.

[9] J. Zou, C. Liu, J. Schutt-Aine, J. Chen, and S-M Kang, "Development of a wide tuning range MEMS tunable capacitor for wireless communication systems," Proc. IEDM, pp. 403-406, December 10-13, 2000.

[10] M. Barbic, J.J. Mock, A.P. Gray, and S. Schultz, "Electromagnetic micromotor for microfluidics applications," Applied Physics Letters, 79(9), 1399-1401 (2001). 